\title{
Colloidal diffusion and hydrodynamic screening near boundaries
}

\author{
Pushkar P. Lele, $\dagger_{\dagger}^{a} \dagger^{a}$ James W. Swan, $\dagger^{a b}$ John F. Brady, ${ }^{b}$ Norman J. Wagner ${ }^{a}$ and Eric M. Furst ${ }^{* a}$ \\ Received 10th December 2010, Accepted 5th May 2011 \\ DOI: $10.1039 / \mathrm{c0sm01466d}$
}

\begin{abstract}
The hydrodynamic interactions between colloidal particles in small ensembles are measured at varying distances from a no-slip surface over a range of inter-particle separations. The diffusion tensor for motion parallel to the wall of each ensemble is calculated by analyzing thousands of particle trajectories generated by blinking holographic optical tweezers and by dynamic simulation. The Stokesian Dynamics simulations predict similar particle dynamics. By separating the dynamics into three classes of modes: self, relative and collective diffusion, we observe qualitatively different behavior depending on the relative magnitudes of the distance of the ensemble from the wall and the inter-particle separation. A simple picture of the pair-hydrodynamic interactions is developed, while many-bodyhydrodynamic interactions give rise to more complicated behavior. The results demonstrate that the effect of many-body hydrodynamic interactions in the presence of a wall is much richer than the single particle behavior and that the multiple-particle behavior cannot be simply predicted by a superposition of pair interactions.
\end{abstract}

\section{Introduction}

The motions of small objects suspended in a viscous medium are strongly coupled by hydrodynamic interactions. ${ }^{1}$ Such interactions arise due to disturbances of the surrounding fluid that viscously propagate in a quasi-steady fashion at small length scales. This small-length-scale-regime is characterized by the Reynolds number $(\operatorname{Re}=\rho U a / \eta \ll 1$, with $\rho$ and $\eta$ the fluid density and viscosity, $U$ the characteristic speed of the particles and $a$ the radius of the particles). In this regime, viscous forces dominate the hydrodynamic resistivity of particles, including colloids, polymer segments and living organisms, such as motile bacteria, which in turn determines the transport properties of materials, such as the diffusivity and viscosity. Thus, hydrodynamic interactions are ubiquitous and important in many soft materials, both natural and man-made, such as suspensions, polymer solutions, slurries and protein solutions.

There is a long history of work examining the effect of hydrodynamic drag due to a nearby wall on the sedimentation velocities of objects. ${ }^{2-5}$ These interactions have also been studied extensively in semi-dilute and concentrated suspensions near

${ }^{a}$ Department of Chemical Engineering and Center for Molecular and Engineering Thermodynamics, University of Delaware, 150 Academy Street, Newark, Delaware, 19716, USA. E-mail: furst@udel.edu; Tel: $+13028310102$

${ }^{b}$ Division of Chemistry and Chemical Engineering, California Institute of Technology, Pasadena, California, 91125, USA

$\uparrow$ Swan and Lele made equal contributions to the work and are considered co-first authors.

† Present address: Harvard Biological Laboratories, Harvard University, 16 Divinity Avenue, Room 3063, Cambridge, MA 02138. surfaces, often termed as quasi-two dimensional suspensions. ${ }^{6-13}$ Likewise, the effect of wall-induced drag on the single particle mobility has been studied in detail using a range of different techniques, including photonic force microscopy, ${ }^{14}$ optical tweezers combined with video microscopy or TIRM (total internal reflection microscopy $)^{15-18}$ and evanescent wave dynamic light scattering (EWDLS). ${ }^{12,19}$ These investigations confirm that single particle diffusivities parallel and normal to the no-slip surface are well approximated by established analytical expressions, ${ }^{20-22}$ and specifically, that the interactions with a no-slip surface decay in strength with the inverse of the distance of the particle center from the wall. Although recent work using blinking optical tweezers has examined the many-body interactions among three ${ }^{23}$ and eight ${ }^{24}$ colloidal particles far from any surfaces, the observation and understanding of many-body hydrodynamic interactions near a no-slip surface is more limited.

In this work, we use Stokesian Dynamics simulations ${ }^{25}$ and blinking optical tweezers to experimentally measure the diffusivity of multi-particle clusters near a single, planar, no-slip surface. We systematically vary the particle-wall separation, $h$, and the inter-particle separation, $r$. Before discussing our results, we first consider the theoretical approaches to solving this manybody problem.

Hydrodynamic interactions in an ensemble of $N$ particles can be quantified in terms of the short-time, ensemble diffusion tensor,

$$
\mathbf{D}=\left.\frac{1}{2} \frac{d}{d t}\langle(\mathbf{x}(t)-\langle\mathbf{x}(t)\rangle)(\mathbf{x}(t)-\langle\mathbf{x}(t)\rangle)\rangle\right|_{t=0},
$$

where $t$ is time and $\mathbf{x}(t)$ represents the trajectories of purely Brownian (otherwise unforced) particles. The diffusion tensor is 
related to the resistance tensor $\mathbf{R}_{F U}$ by the Einstein relation, $\mathbf{D}=$ $k T \mathbf{R}_{F U}^{-1}$, where $k T$ is the thermal energy. The resistance tensor depends only on the relative configuration of the ensemble (i.e. the inter-particle separations), the position of the ensemble relative to any macroscopic boundaries (e.g. a no-slip plane wall), the particle sizes and the fluid viscosity. In the simplest approximation of the hydrodynamics of colloidal particles above a no-slip boundary the particles are treated as point forces. Then the velocity field at $\mathbf{x}$ due to a point force, denoted $\mathbf{f}$, in the fluid at $\mathbf{y}$ is

$$
\mathbf{u}(\mathbf{x})=\mathbf{G}(\mathbf{x}, \mathbf{y}) \cdot \mathbf{f} .
$$

Here, $\mathbf{G}(\mathbf{x}, \mathbf{y})$ is the Green's function for Stokes flow above a rigid, no-slip plane wall. Without a wall, this function is simply the Stokeslet ${ }^{26}$

$$
\mathbf{J}(\mathbf{r})=\frac{1}{8 \pi \eta r}(\mathbf{I}+\hat{\mathbf{r}} \hat{\mathbf{r}})
$$

where $\mathbf{I}$ is the idem tensor, $r=|\mathbf{x}-\mathbf{y}|$ is distance between the source and field points and $\hat{\mathbf{r}}=(\mathbf{x}-\mathbf{y}) / r$ is the unit vector connecting these points. In order to satisfy the no-slip condition on the plane wall, additional hydrodynamic flows must be generated by the point force so that

$$
\mathbf{G}(\mathbf{x}, \mathbf{y})=\mathbf{J}(\mathbf{x}-\mathbf{y})+\mathbf{J}_{W}(\mathbf{x}, \mathbf{y})
$$

and $\mathbf{J}_{W}(\mathbf{x}, \mathbf{y})=-\mathbf{J}(\mathbf{x}-\mathbf{y})$ when $\mathbf{x}$ is a point on the boundary. These additional flows may be determined via the method of images such that ${ }^{27}$

$$
\begin{gathered}
\mathbf{J}_{w}(\mathbf{R}, h)=-\mathbf{J}(\mathbf{R})+h^{2} \nabla_{x}^{2} \mathbf{J}(\mathbf{R}) \cdot\left(\mathbf{I}-2 \mathbf{e}_{\perp} \mathbf{e}_{\perp}\right) \\
-2 h\left[\left(\mathbf{I}-2 \mathbf{e}_{\perp} \mathbf{e}_{\perp}\right) \cdot \nabla_{x} \mathbf{J}(\mathbf{R}) \cdot \mathbf{e}_{\perp}\right]^{T},
\end{gathered}
$$

where $\mathbf{R}=\mathbf{r}+2 h \mathbf{e}_{\perp}, h$ is the height of the point force above the no-slip boundary and $\mathbf{e}_{\perp}$ is the vector normal to the boundary. One striking consequence of this function is that while the Stokeslet decays in strength as $r^{-1}$, the magnitude of the velocity field due to a point force above a wall may decay as $r^{-2}$. The change from $r^{-1}$ to $r^{-2}$ or in a certain case $r^{-3}$-when the particles are at exactly the same height above the wall (see the appendix) - is termed hydrodynamic screening and is the result of the fluid satisfying the no-slip condition on the plane wall. This may be illustrated via the hydrodynamics of only two particles above a no-slip surface (see Fig. 1).

In Fig. 1, we plot the self, relative and collective diffusivities parallel to the wall of a pair of particles (numbered 1 and 2 less the parallel diffusivity of a single, isolated particle above that wall, denoted $D_{\|}$. This single particle diffusivity depends on the distance of the particle from the wall and expressions for this quantity are well known. ${ }^{22}$ The self diffusive modes correspond to the forcing of a single particle in the pair along $\hat{\mathbf{r}}$, (i.e. $\left.D_{\text {self }}=\hat{\mathbf{r}} \cdot \mathbf{D}^{11} \cdot \hat{\mathbf{r}}\right)$ while the relative and collective modes correspond to the forcing of both particles (in the directions $\hat{\mathbf{r}}$ and $-\hat{\mathbf{r}}$, and $\hat{\mathbf{r}}$ and $\hat{\mathbf{r}}$, respectively). These diffusivities are:

$$
D_{\text {relative }}=\hat{\mathbf{r}} \cdot\left(\mathrm{D}^{11}-\mathrm{D}^{12}\right) \cdot \hat{\mathbf{r}}
$$

and

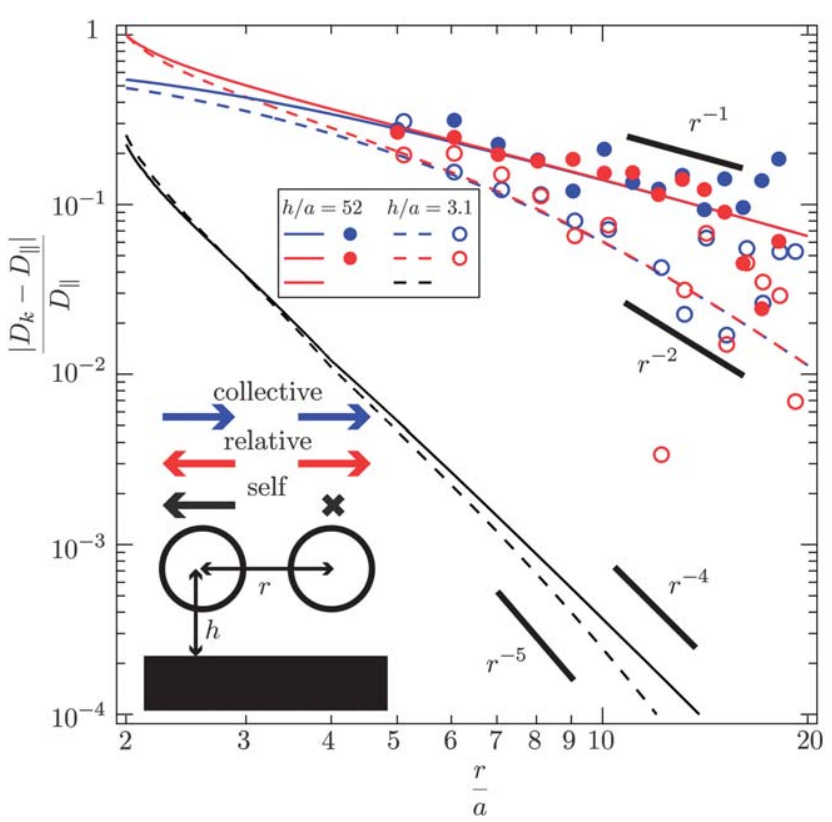

Fig. 1 The additional hinderance (self and relative) and enhancement (collective) of various parallel diffusive modes due to hydrodynamic interactions between a pair of particles is plotted as a function of the separation between the particles and the height of the pair above a plane wall. The solid lines and filled symbols correspond to $h / a=52$ while the dashed lines and open symbols correspond to $h / a=3.1$. The points are experimental data from Dufresne et al. who measured the dynamics of particle pairs above a single plane wall using optical tweezers. ${ }^{30}$ The lines are the results of Stokesian Dynamics simulations.

$$
D_{\text {collective }}=\hat{\mathbf{r}} \cdot\left(\mathrm{D}^{11}+\mathrm{D}^{12}\right) \cdot \hat{\mathbf{r}}
$$

respectively. The self and relative diffusion are retarded by hydrodynamic interactions (i.e $D_{\text {self }}, D_{\text {relative }}<D_{\|}$) while the collective diffusion is enhanced (i.e. $D_{\text {collective }}>D_{\|}$).

The diffusivity tensor $\mathbf{D}$ has elements, $\mathbf{D}^{\alpha \beta}$, corresponding to the coupling of diffusion of particle $\alpha$ with diffusion of particle $\beta$. For instance, if $\alpha$ and $\beta$ are the same, then this element of $\mathbf{D}$ corresponds to self-diffusion. Note though, even $\mathbf{D}^{\alpha \alpha}$ includes many-body hydrodynamic interactions as will be illustrated shortly. By treating the particles as point forces that must move with the fluid, the parallel components of $\mathbf{D}^{\alpha \alpha}$ are simply $k T$ $\left[(6 \pi \eta a)^{-1}+\mathbf{e}_{\|} \mathbf{e}_{\|}: \mathbf{J}_{W}\left(\mathbf{x}^{\alpha}, \mathbf{x}^{\alpha}\right)\right]$ where $\mathbf{x}^{\alpha}$ is the position of particle $\alpha$. Similarly the parallel components of $\mathbf{D}^{\alpha \beta}$ are $k T \mathbf{e}_{\|} \mathbf{e}_{\|}: \mathbf{G}\left(\mathbf{x}^{\alpha}, \mathbf{x}^{\beta}\right)$ for point-particles (note that ":" represents the double-dot-product operation, i.e $\left.\mathbf{A}: \mathbf{B}=\sum_{i, j=1}^{3} A_{i j} B_{i j}\right)$. This point-particle approximation is insufficient in many ways, which are addressed throughout the article. More complete descriptions of the hydrodynamics requiring little additional computational effort have been developed including the Stokesian Dynamics simulations employed herein. ${ }^{25}$ The point force/particle approximation gives just the leading order behavior (up to $r^{-2}$ and $h^{-1}$ ) for widely separated particles far from the wall $(r, h \gg a)$. However, this is the proper starting point for developing a more rigorous theory. Beginning ad hoc with hydrodynamic interactions derived for other purposes (e.g. the Rotne-Praeger tensor ${ }^{28}$ ) may lead to diffusivity tensors that do not satisfy some fundamental 
constraints intrinsic to low-Reynolds-number flows. ${ }^{29}$ Namely, the diffusivity tensor must be symmetric and positive definite.

In Fig. 1, the results of Stokesian Dynamics simulations (lines) are compared with the experiments of Dufresne et al. (points), for particle pairs set distances $h / a=3.1$ and $h / a=52$ above the wall. ${ }^{30}$ Notice that the dependence on the inter-particle separation is distinctly different in each of these cases. When $a \ll r \ll h$, the particles are close enough that hydrodynamic interactions with the wall are irrelevant, and the relative and collective interactions decay as $r^{-1}$. While for $h \ll r$, the hydrodynamic interactions with the wall are significant and the relative- and collective-modescaling switches to $r^{-2}$. The $r^{-2}$ interaction is characteristic of force dipoles, and indeed, through the method of images, we can see that $\mathbf{G}(\mathbf{x}, \mathbf{y})$ has a dipolar character when $h \ll r$; to leading order there is an image force, $-\mathbf{J}(\mathbf{R})$, below the wall.

The retardation of the self-diffusive mode has a stronger decay than either the relative or the collective modes as one particle is forced and the other must remain force free. In order for the no-slip boundary condition to be satisfied on the surface of the unforced particle, the force density on the surface of that particle must change so that the unforced particle acts, to a first approximation as a force dipole or stresslet itself. Therefore, where the hydrodynamic interaction for the relative and collective modes was direct through the forcing of both particles, the hydrodynamic interaction for the self-diffusive mode is indirect and comes about through reflection of the disturbance generated by the forced particle. This behavior cannot be captured by the point force/particle model of hydrodynamic interactions. Rather, higher order moments of the force density on the particle surfaces are necessary. Still, the effect of hydrodynamic screening is evident as the self-interaction changes in rate of decay from $r^{-4}$ to $r^{-5}$ as the forced particle is brought very near the wall. It is this interaction that characterizes the self-diffusivity in suspensions as well; thus, hydrodynamic screening may be important in setting the dynamics of many particles near a boundary.

In order to examine the many-body hydrodynamic interactions of particles parallel to a no-slip boundary, we construct an ensemble of seven particles residing on the vertices and in the center of a hexagon. We then measure the mean-squared displacement of these particles parallel to the wall experimentally and computationally to quantify the enhancement and retardation of various diffusive modes. We find quantitative agreement between the experimentally determined diffusivities and those calculated via Stokesian Dynamics simulations. This simultaneously validates the experimental procedure and the theory employed in developing Stokesian Dynamics. Before discussing our results further, we describe the statistical procedures and experimental and simulation methods employed in this study.

\section{Methodology}

\subsection{System}

Seven spherical particles are arranged in a hexagonal configuration (six particles on the vertices, one particle in the center) with the plane of the hexagon parallel to a no-slip wall (illustrated in Fig. 2). The side length of the hexagon is equivalent to nearest neighbor inter-particle separation, and this quantity is varied experimentally in the range: $2.6<r / a<4$. 6 . The distance

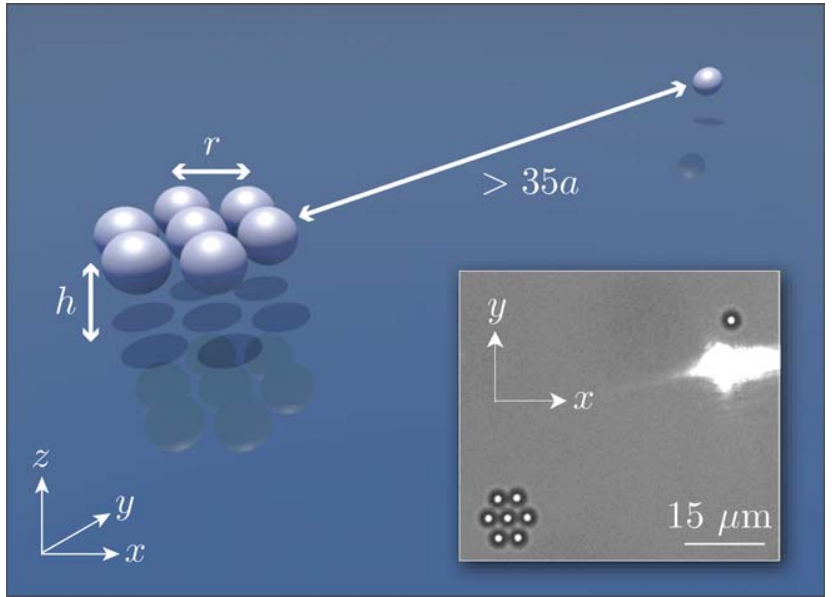

Fig. 2 The diffusion of seven spherical particles positioned on the vertices and in the center of a hexagon with side $r$ and height $h$ above a plane wall is studied experimentally and via simulation. This diagram reflects the relative configuration of particles and the wall as well as the existence of a reference particle in the experiments used to determine precisely the distance of the particles from the wall. Bright field microscopy images the configuration from the top down (see the inset).

between the wall and the ensemble is varied with: $1.1<h / a<72$. The particles are released from their initial hexagonal configuration and Brownian motion causes the configuration to distort. We measure the $N$-body diffusion tensor dynamically as

$$
\mathbf{D}^{\alpha \beta}=\frac{\left\langle\left(\mathbf{x}^{\alpha}(\tau)-\left\langle\mathbf{x}^{\alpha}(\tau)\right\rangle\right)\left(\mathbf{x}^{\beta}(\tau)-\left\langle\mathbf{x}^{\beta}(\tau)\right\rangle\right)\right\rangle}{2 \tau},
$$

where $\mathbf{x}^{\alpha}(\tau)$ is the change in position of particle $\alpha$ from its place in the initial configuration after the lag time, denoted $\tau$, which is chosen to be short relative to the single particle diffusive time-scale $\left(a^{2} / D_{\|}\right)$. The angle brackets reflect an average over many realizations (experimental trajectories) that begin with the particles in the hexagonal configuration. For a small enough $\tau$ and enough realizations, the short-time dynamics of the ensemble are measured and the diffusivity, $\mathbf{D}$, corresponds to that arising from inversion of the hydrodynamic resistance tensor for the initial hexagonal configuration. The trajectories are measured experimentally and computed by dynamic simulation for 1500 and 10000 realizations, respectively. From this the mean-squared displacements are calculated yielding the $N$-body diffusivity tensor.

This same hexagonal arrangement is studied systematically via Stokesian Dynamics simulation at two fixed inter-particle separations while varying continuously height above the wall and at two fixed heights while varying continuously the inter-particle separation. In this particular case, rather than calculate the diffusion tensor dynamically as done for comparison with the experiments, we simply compute $\mathbf{D}=k T \mathbf{R}_{F U}^{-1}$ as calculation of the hydrodynamic resistance is the fundamental purpose of Stokesian Dynamics. In this way, no averaging over realizations is required and data with zero statistical noise generated.

\subsection{Experimental}

Our sample cell consists of a glass cover slip and microscope slide with cover slides used as spacers to create a gap of approximately 
$160 \mu \mathrm{m}$. The glass surfaces are cleaned overnight in a freshly prepared solution of sulfuric acid and an inorganic oxidizer (Nocrhomix, Cat. no. 19-010, Godax Laboratories, Cabin John, $\mathrm{MD})$. Prior to all experiments, we treat the cell with a plasma cleaner (PDC-32G, Harrick Plasma, Ithaca, NY) before introducing the colloidal suspension by capillary action. The ends of the cell are sealed using an ultraviolet curing adhesive (NOA 81, Norland Products, Cranbury, NJ).

Seven-particle clusters are trapped by custom-built holographic optical tweezers. The particles are monodisperse polystyrene latex beads with radius $a=1.41 \pm 0.1 \mu \mathrm{m}$ (Polysciences, Warrington, PA). The optical traps are generated by a nearinfrared ytterbium fiber laser (YLR-10-1070-LP, IPG Photonics, Oxford, MA) with vacuum wavelength $\lambda=1070 \mathrm{~nm}$, and a spatial light modulator (Boulder Nonlinear Systems, Lafayette, $\mathrm{CO}$ ) controlled by a workstation using a commercial software package (HOTAPI, Arryx, Chicago, IL). A microscope (Axiovert, Carl Zeiss, Thornwood, NY) equipped with a high numerical water immersion objective $(63 \times, 1.2$ numerical aperture $\mathrm{C}$-apochromat) serves as both the optical trapping and imaging system.

Particles are arranged on a hexagonal lattice with spacing $r$, as shown in Fig. 2 in a single plane close to the glass coverslip. We simultaneously trap a single particle at a distance more than $35 a$ away from the cluster. This reference particle is kept far enough away that the interaction with the individual particles in the cluster is insignificant. Its mobility is a measure of the limiting behavior of individual particles in the cluster at large interparticle separations. To accurately set the focal plane height above the coverslip surface, we use a feedback-controlled piezo objective nanopositioner (PIFOC, P-721.CDQ, Physik Instrumente, Karlsruhe, Germany). As a direct verification of the height, we also simultaneously create an empty "guide point" optical trap offset vertically by the spatial light modulator to focus on the surface. From the intensity scattered from the guide trap, it is possible to independently verify the focal height and locate particles at reproducible distances from the surface. The reference particle diffusivity also provides an accurate confirmation of the absolute distance of the cluster from the surface when compared with known expressions for $D_{\|}$. In Fig. 3, we compare experimental measurements of the single particle, shorttime diffusivity parallel to the wall with the exact expression. ${ }^{22}$

We generate several thousand trajectories by periodically shuttering the laser beam using a signal from a function generator with a $1: 3$ duty cycle (DS-360, Stanford Research Systems, Sunnyvale, CA) to control a mechanical shutter (DSH-10, Electro-Optical Products, Ridgewood, NY). Particle dynamics are recorded using a high-speed digital camera (Phantom v5.1, Vision Research, Wayne, NJ) and the individual particle trajectories are obtained from the digital images using standard particle tracking algorithms. ${ }^{31} \mathrm{~A}$ co-aligned helium neon laser (vacuum wavelength $\lambda=632.8 \mathrm{~nm}$, CVI Melles Griot, Albuquerque, NM) is used to generate a bright spot in the video image in order to track the shutter state. The beam is shuttered for $0.6 \mathrm{~s}$, or approximately $1 / 20$ th of the characteristic particle diffusion time, $6 \pi \eta a^{3} / k T$ to ensure that the particles are re-trapped during each cycle. The measurements are carried out in dilute salt solutions to screen long-range electrostatic interactions between the particles and the sample wall.

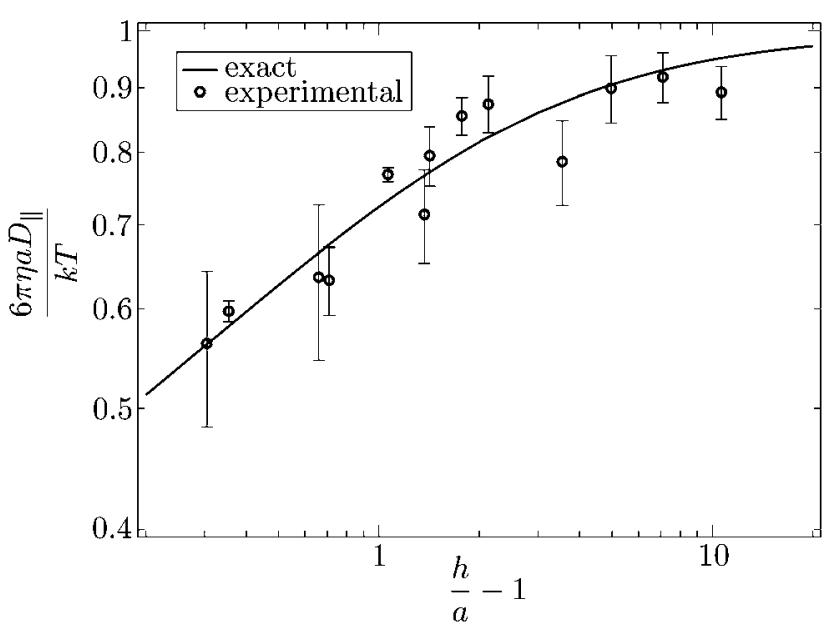

Fig. 3 The experimental measurement of the parallel, short-time diffusivity of a single particle above the wall. The method for measuring the height of a single particle above the wall and corresponding measurements of the parallel diffusivity agree with the exact, analytical expression for the same quantity.

\subsection{Computational}

A full description of the Stokesian Dynamics technique for particles near a plane wall is put forth in the article by Swan and Brady ${ }^{25}$ - it is beyond the purview of this article. However, some features of Stokesian Dynamics highlight key physics in the hydrodynamic interactions of particles regardless of any boundary geometry. As two spherical particles approach each other along their line of centers or a spherical particle approaches normal to a no-slip plane wall, tremendous forces must be applied to squeeze fluid out of the thin gap between the surfaces. These forces, sometimes referred to a lubrication forces, dominate the hydrodynamic interactions of nearly touching and immersed surfaces. ${ }^{26}$ For instance, pushing a spherical particle into a plane wall requires a force proportional to $(h-a)^{-1}$ when $h-a \ll 1$. Where the point force/particle model of hydrodynamic interactions captured only the most coarse or long-ranged interactions, the lubrication forces describe only the near-field interactions among particles. Because lubrication forces are so strong and arise from the flow of fluid in the thin gap between surfaces undergoing relative motion, they may be approximated to a good degree as pair-wise additive. The same is not true of the far-field hydrodynamic interactions which are many-bodied.

Stokesian Dynamics accounts for the difference in the character of these two interaction regimes (near-field and far-field) by computing the hydrodynamic forces due to each interaction independently subject to the constraint that the particles move in a self-consistent manner. That is, the sum of the hydrodynamic force due to the far-field interactions, proportional to $k T \mathbf{D}_{f f}^{-1}$ where $\mathbf{D}_{f f}$ is the diffusivity tensor arising from a far-field hydrodynamic theory (the simplest approximation would be the diffusivity of point particles), and the hydrodynamic force due to the near-field interactions (easily computed via lubrication theory $)^{22}$ balances any external forces on the particles (e.g. gravity or Brownian forces). However, the particle velocities to which the hydrodynamic forces (both near-field and far-field) are linearly proportional must be the same. The 
constraint that the hydrodynamic forces balance any external forces and that the particle velocities are consistent across interaction regimes is sufficient to determine the particle motion arising from those external forces. ${ }^{1}$

Whereas the near-field interactions are pair-wise additive, the far-field interactions are many-bodied. It has been shown that the inversion of even the point force/particle approximation for $\mathbf{D}_{f f}$ will give rise to a many-body hydrodynamic resistance. ${ }^{32}$ This inversion is equivalent to a method of reflections approach to determining the hydrodynamic interactions. ${ }^{26}$

\section{Results and discussions}

We measure several different diffusive modes characterized by the diffusivity denoted,

$$
D_{k}=\mathbf{X}_{k} \cdot \mathbf{D} \cdot \mathbf{X}_{k}
$$

where $\mathbf{X}_{k}$ is a unit vector characterizing the particular diffusive mode $k$. The normal modes (i.e the exact eigenvectors of the diffusion tensor) might be used for trajectory analysis. However, these modes (even for a pair of particles) are a function of the inter-particle separation and distance of the particles from the wall. This makes physical interpretation of the corresponding diffusivities problematic. Instead, mutually orthogonal physical modes such as those employed in section 1 provide insight into the relative and collective motions of the particles. The directionality of these modes is independent of the inter-particle separation and height of the particles above the wall. Ten such modes for the hexagonal configuration are pictured in Fig. 4 where modes 1-4 refer to motion of a single particle in the ensemble (self), modes 5-8 are eigenvectors of the diffusion tensor (projected in two dimensions) arising from the six fold symmetry of the ensemble (relative), and 9-10 correspond to sedimentation of the ensemble (collective). The arrows in the figure correspond to vector elements of $\mathbf{X}_{k}$ so that $\mathbf{X}_{k}^{\alpha}$, the element of $\mathbf{X}_{k}$ associated with particle $\alpha$, is a vector parallel to the

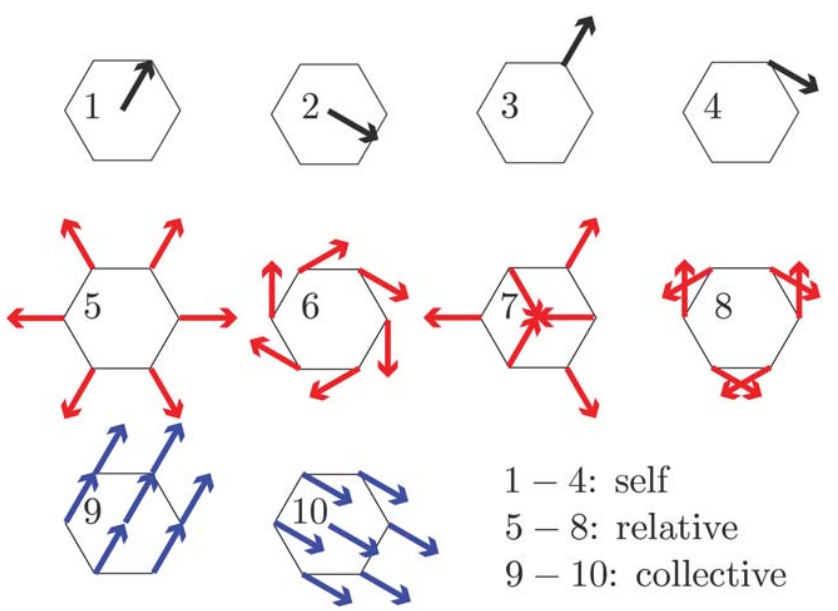

Fig. 4 The diffusive modes studied via experiment and simulation are pictured. The arrows reflect the vectors $\mathbf{X}_{k}$ for each mode $k$ where 1-4 correspond to self-diffusion, modes 5-8 correspond to relative diffusion and 9-10 correspond to collective diffusion. The particles reside at the vertices and in the center of the hexagon. particular arrow for particle $\alpha$ in mode $k$. The analogy with the modes illustrated in Fig. 1 is evident. Note, that the relative and collective modes are orthonormal as well so that $\mathbf{X}_{j} \cdot \mathbf{X}_{k}=\delta_{j k}$ just as they were in the pair problem.

These modes may be thought of in at least two different ways. Consider that were, $\mathbf{F}_{k}=\mathbf{X}_{k}$, a force on the particles, then the quantity:

$$
\frac{D_{k}}{D_{\|}}=\frac{\mathbf{F}_{k} \cdot \mathbf{U}_{k}}{D_{\|} / k T},
$$

where $\mathbf{U}_{k}=\mathbf{R}_{F U}^{-1} \cdot \mathbf{F}_{k}$, is the ratio of the rate of energy dissipated by the fluid due to forcing of the particles in this manner to the rate of energy dissipated by a single particle forced with strength $\sqrt{\mathbf{F}_{k} \cdot \mathbf{F}_{k}}$ parallel to the wall (of course, $\mathbf{X}_{k}$ is normalized so that the dot product is unity). This is just a restatement of the fluctuation-dissipation theorem. Alternatively, the diffusive modes measure the speed of Brownian motion along particular directions. In this way, $D_{k}$ measures the rate of fluctuations in the particle positions, but only those fluctuations consistent with the arrows depicted in the Fig. 4. We are able to measure all 196 elements of the 2-D projection of $\mathbf{D}\left[(7 \text { particles } \times 2 \text { directions })^{2}\right]$. This data is difficult to rationalize physically, however, so that we compare just ten physical modes of diffusion. A full eigendecomposition of the diffusion tensor was conducted by Lele ${ }^{33}$ and found similar quantitative agreement between the experiments and Stokesian Dynamics simulations.

For a range of inter-particle separations and heights of the ensemble above the wall, we measured these diffusivities experimentally and computed them dynamically via Stokesian Dynamics. Fig. 5 compares the experimental and computational results, showing very good agreement. Here we have plotted the quantity $\left(D_{k}-D_{\|}\right) / D_{\|}$in order to highlight the effect of the

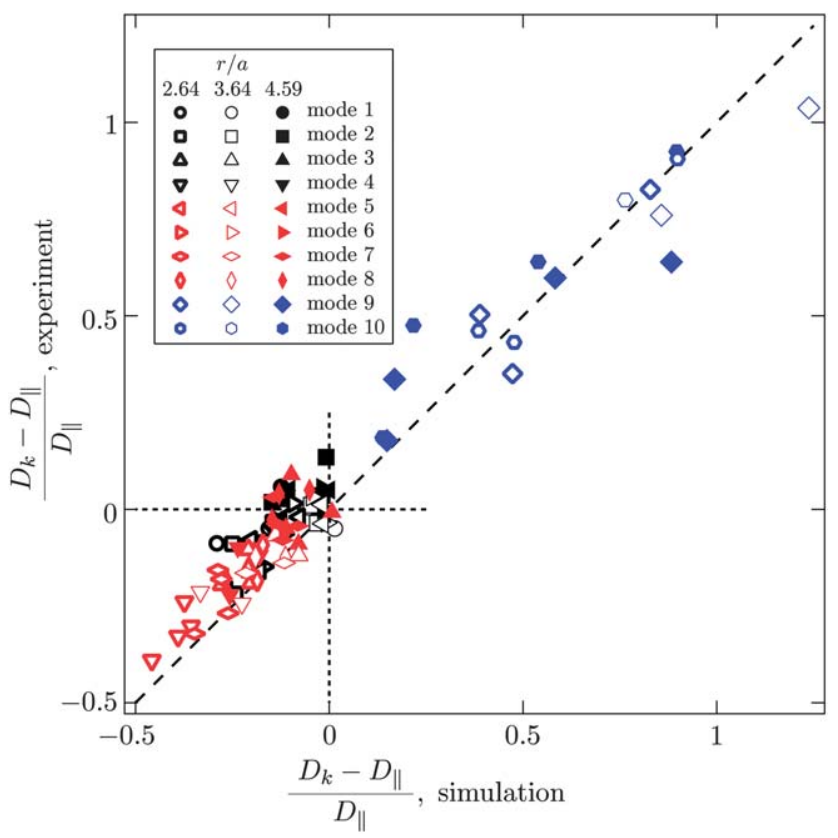

Fig. 5 The diffusive modes were measured experimentally and calculated dynamically via Stokesian Dynamics simulation for configurations with $r / a=2.64: h / a=1.1,1.2,1.89,72 ; r / a=3.64: h / a=6,14.48 ; r / a=4.59: h / a=$ $1.1,1.32,2.74,8.66$. The lag time in all cases was $\tau k T / 6 \pi \eta a^{3}=0.016$. 
inter-particle spacing. Each of these ensembles is positioned at a different height above the wall, and yet the self, relative and collective modes segregate clearly when compared on this basis. The hinderance to the self and relative modes and the enhancement of the collective modes is strongest when the particles are closest together. These effects weaken as the inter-particle spacing is increased.

Additionally, the diffusivity corresponding to a particular hexagonal configuration may be computed exactly as the inverse of the hydrodynamic resistance tensor (i.e. $\mathbf{D}=k T \mathbf{R}_{F U}{ }^{-1}$ ). This enables prediction of the diffusive modes for a continuous range of inter-particle separations and heights. Conversely, generating consistent results for one configuration from dynamic simulations requires thousands of realizations during which the hydrodynamic resistance is calculated at each time as the configuration evolves. In Fig. 6, we compare the results of dynamic simulations with lag time $\tau k T / 6 \pi \eta a^{3}=0.016$ to the diffusion tensor calculated exactly for the same configurations. The lag-time based diffusivity measurement features some quantitative changes in several of the diffusive modes as well as a qualitative change in mode 6 . Differences in the diffusivities themselves $\left(D_{k}\right)$ are relatively small $(<10 \%)$, but since we are removing the single particle diffusivity from the many-body diffusion modes, errors are more pronounced. These differences between the exact calculation and the lag-time measurements are easily understood, however.

Note that the mean velocity of each of the particles in the hexagonal configuration,

$$
\frac{d}{d t}\langle\mathbf{x}(t)\rangle=\langle\nabla \cdot \mathbf{D}\rangle
$$

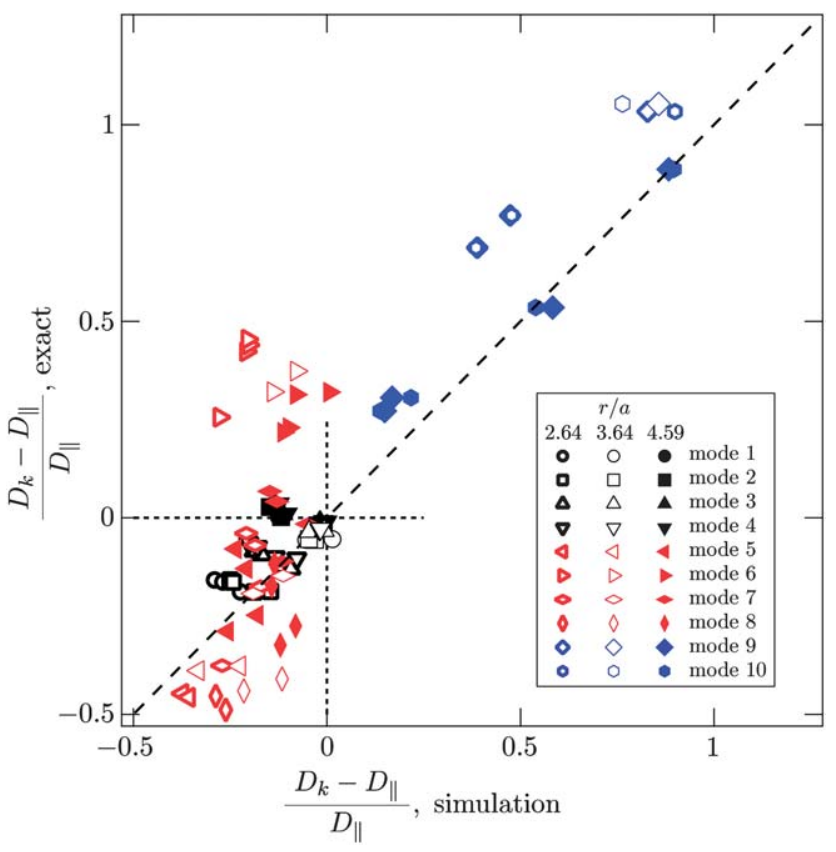

Fig. 6 The diffusive modes were calculated via Stokesian Dynamics simulation for configurations with $r / a=2.64$ : $h / a=1.1,1.2,1.89,72$; $r / a=3.64: h / a=6,14.48 ; r / a=4.59: h / a=1.1,1.32,2.74,8.66$. The lag time in all cases was $\tau k T / 6 \pi \eta a^{3}=0.016$. is non-zero over the course of the experiments and simulations. The non-zero velocity is referred to as hydrodynamic drift and arises precisely because the diffusion tensor depends on the relative configuration of the particles. $\S$ The mean drift causes the particle configuration to broaden and move away from the wall. The broadening leads to weaker hydrodynamic interactions between the particles so that the lag-time data: $\left(D_{k}-D_{\|}\right) / D_{\|}$, are shifted toward zero (see Fig. 6). This appears to be the case for many of the modes $(5,7,9,10)$.

There is another source of error in the lag-time measurement which is independent of the mean drift. Within an individual dynamic realization, the configuration of the particles fluctuates about the trajectory described by the mean drift. Consequently, the diffusivity measured dynamically corresponds to an average over these distorted configurations. This distortion of the configuration occurs both in-plane and out-of-plane so that the rotationally symmetric mode (i.e. mode 6) is affected in dramatic fashion. For mode 6 and from the perspective of the static configuration, each particle entrains and is entrained by its neighbors so that ring-like-diffusion is enhanced by hydrodynamic interactions. However, as the configuration of the particles fluctuates and particles fall out of the plane of the hexagon, the degree of entrainment is reduced so that enhancement becomes retardation. Evidently, we do not measure the particle trajectories in either experiment or simulation over a short enough lagtime to minimize the configurational fluctuations and capture this behavior. We have observed, however, that as the lag-time is reduced, $\left(D_{6}-D_{\|}\right) / D_{\|}$grows more positive supporting this preceding rationale.

Fig. 7 shows a direct comparison between the exact diffusion modes obtained via Stokesian Dynamics simulations and the results of blinking optical tweezers experiments for the case that $r / a=2.64,4.59$ and for a variety of heights. The qualitative agreement between the simulation and experiments is very good. The trends as a function of the height above the wall meet expectations: hydrodynamic screening suppresses the enhancement/retardation of all the diffusive modes as a configuration of particles is brought nearer to the wall. All of the modes, excluding mode 6 , decay monotonically. Similarly all the modes, except mode 7 when $r / a=4.59$, have a single sign.

The same calculations are analyzed to examine the behavior of two ensembles for which $h / a=3.1$ and $h / a=52$ by systematically varying the inter-particle separation. These simulations are the many-body analog of Fig. 1. Again, rather than compute the diffusivity tensor for the ensemble dynamically via trajectory analysis, it is computed directly for a continuous range of $r / a$. The results are depicted in Fig. 8. When the diffusive modes for a pair of particles above a plane wall at these two heights were examined, the collective mode was enhanced and the self and relative modes were retarded by hydrodynamic interactions for all $r / a$. A similar picture emerges from study of these hexagonal configurations. The collective and self modes are enhanced and retarded, respectively, regardless of the inter-particle separation

$\S$ The point-force/point-particle model of hydrodynamic interactions produces zero mean drift. In fact, the same is true of the higher order Rotne-Praeger approximation that accounts for the finite size of the particles. ${ }^{28}$ A higher order description accounting for at least the torque and stresslet is needed to obtain the mean drift. 


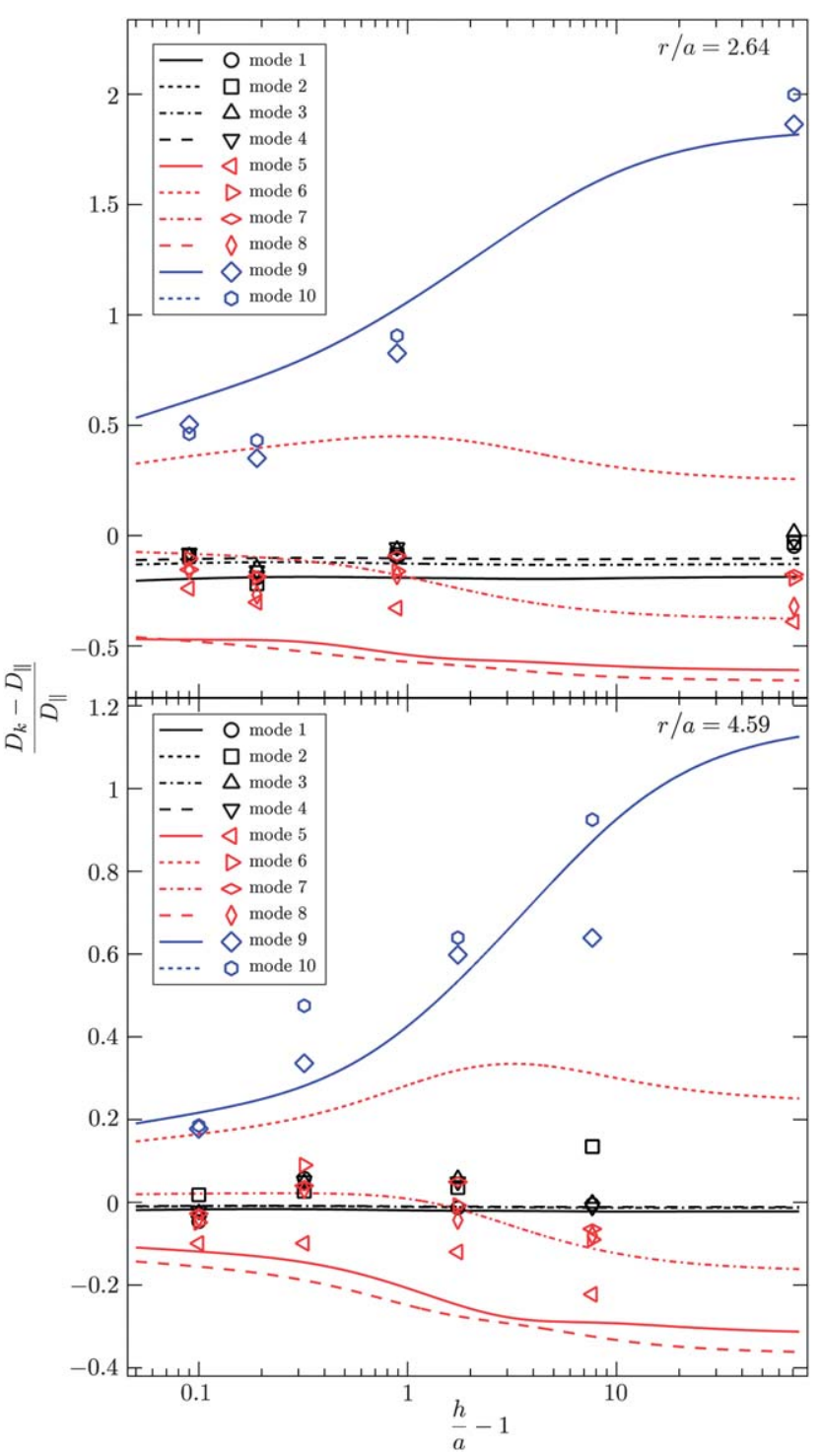

Fig. 7 The diffusive modes for hexagonal configurations at many heights above a no-slip plane wall were measured experimentally (open symbols) and computed via Stokesian Dynamics simulations (lines). These configurations correspond to inter-particle separations of $r / a=$ 2.64 and $r / a=4.59$.

or height above the wall. In fact, the degree of enhancement or retardation decays just as with particle pairs (as $r^{-1}$ or $r^{-2}$ for collective and $r^{-4}$ or $r^{-5}$ for self with $r \ll h$ or $r \gg h$ ). The relative modes all decay in the same manner as the collective modes; however, only two of the relative modes: 5 and 8, are purely retarded by hydrodynamic interactions.

Mode 6 is enhanced regardless of the inter-particle separation for both heights above the wall and reflects the diffusive rotation of the ensemble about the center particle. This mode is termed relative because the vectors along which the mean-squared displacement is projected are not parallel (see Fig. 4). However, the projection of the vectors corresponding to neighboring particles is positive. If we focus on a single pair of neighboring vertex particles alone, we see that diffusion along those vectors is a superposition of the relative and collective modes described for

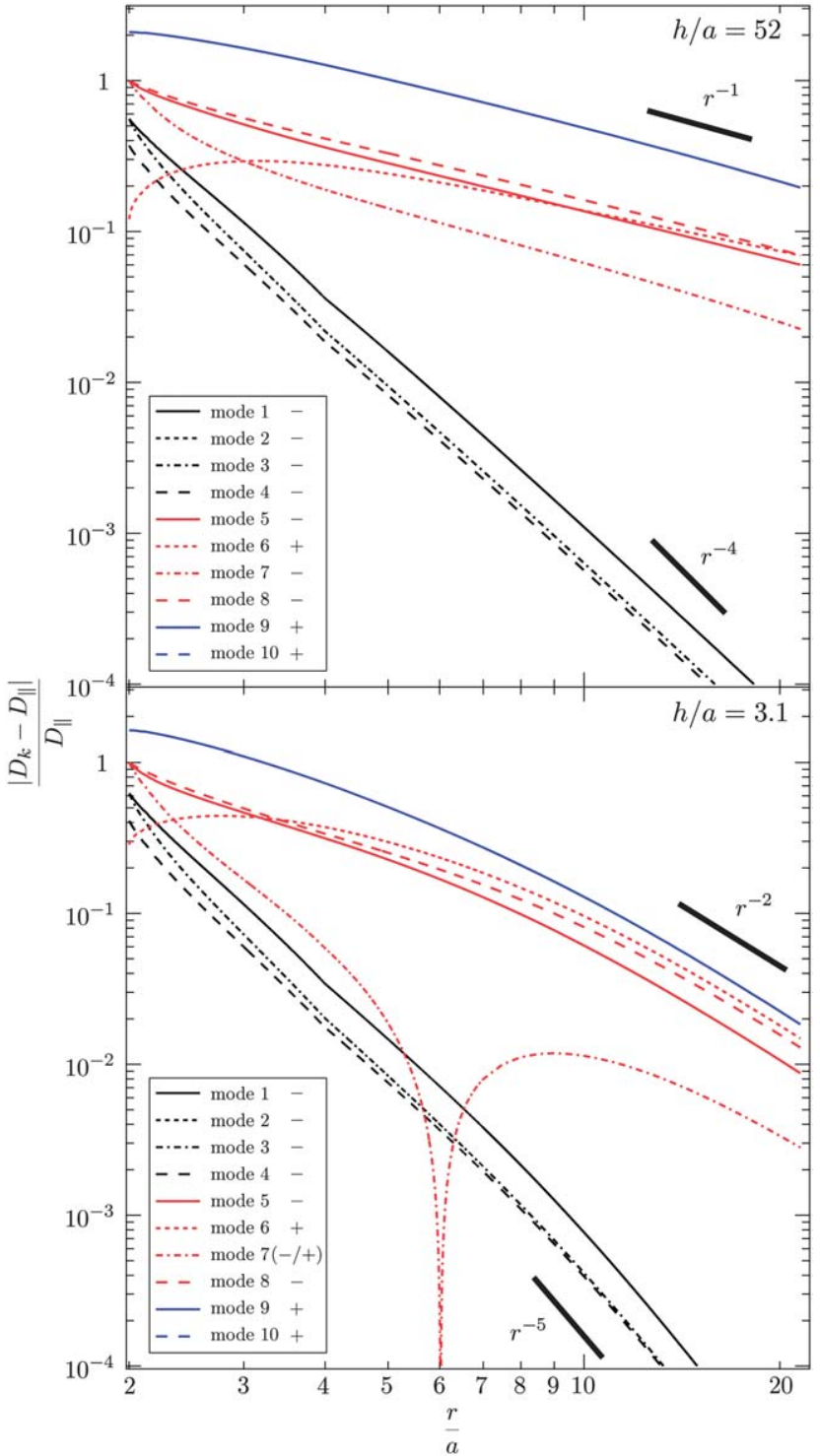

Fig. 8 The diffusive modes for hexagonal configurations at $h / a=52$ (top) and $h / a=3.1$ (bottom) above a no-slip plane wall were computed via Stokesian Dynamics. As it is the absolute value of the difference between the single particle diffusivity and the diffusive mode, each mode has a corresponding + or - indicating whether the difference was positive (enhancement) or negative (retardation).

the particle pair. Therefore, as each particle entrains the particle moving behind it, the diffusion in this mode is enhanced by the hydrodynamic interactions among the particles. Mode 7 exhibits only retardation when the ensemble is far from the wall $(h / a=52)$ for all inter-particle separations. Near the wall, however, this mode is retarded as well but for small inter-particle separations $(r / a<6)$ only. For larger inter-particle separations, this relative mode is enhanced because of a particle-wall type hydrodynamic interaction. That is, the additional flow fields due to motion in the presence of the nearby wall are of sufficient strength to overcome the retarding particle-particle hydrodynamic interactions. These relative modes are especially interesting because they reflect various coordinated motions which could be relevant for cage diffusion in dense suspensions. It is reasonable to infer from Fig. 8 
that mode 7 becomes enhanced at smaller inter-particle separations as $h / a$ approaches unity. As a result, this particular mode of cage diffusion in dense suspensions may also be enhanced which would lead to faster particle dynamics near boundaries.

\section{Conclusions}

The dynamics of small ensembles of colloidal particles near a no-slip surface are studied with the goal of better understanding the influence of particle-wall hydrodynamic interactions in suspension mechanics. The hydrodynamic interactions between particles based on the point force/particle (a.k.a. Oseen-Burgers) description combined with the method of images are shown to be insufficient to describe all the dynamics as demonstrated by the additional hinderance to the self-diffusive modes for just a pair of particles. Instead, the full diffusion tensor computed via Stokesian Dynamics accounts for this behavior. The correlated motion of the particles was evident in the behavior of the self, relative and collective diffusive modes, which are influenced by varying the particle center-to-center separations as well as the distance from the wall. However, the self-diffusive modes have a stronger dependence on the inter-particle separation than can be accounted for by the simple point force model of colloid motion. Over the range of $r / a$ we examined for a hexagonal ensemble of particles, we found that all the self-diffusive modes decayed as either $r^{-4}$ or $r^{-5}$ for $r \ll h$ and $r \gg h$, respectively. Similarly, all the relative and collective modes of diffusion decay as either $r^{-1}$ or $r^{-2}$ under each of the same conditions. However, where we observed that all collective modes of diffusion are enhanced by hydrodynamic interactions while self and relative modes are retarded for a pair of particles regardless of the height above the wall, the same was not true of the hexagonal ensemble. Depending on the height above the wall, one relative diffusive mode (6 in Fig. 4) was enhanced by the interactions regardless of the inter-particle spacing and height above the wall. Similarly, another relative mode (7 in Fig. 4), was either enhanced or retarded when the ensemble was near the wall depending on the inter-particle spacing and was purely retarded when the ensemble was far from the wall. The behavior of mode 6 is the result of many-body (particle-particle) hydrodynamic interactions while the behavior of mode 7 is evocative of additional many-body, particle-wall-particle, hydrodynamic effects.

While this study focused on an isolated cluster of particles near a wall, the same methodology should be applicable to confined suspensions. A similar analysis of two-dimensional diffusion modes is possible because in dense suspensions near a boundary the particles form transversely parallel lamellae within at least two diameters of the wall. Do the same predictions regarding enhancement or hinderance of diffusive modes hold, or do the surrounding and even intervening particles further screen the hydrodynamic interactions among a cluster and alter the behavior? A deeper understanding of the role of hydrodynamic interactions in governing rate processes such as the formation and melting of colloidal crystals and glasses may be developed through the answers to such questions.

\section{Appendix}

As a final note, it is a quirk of the Stokes equations that for two, widely separated particles residing exactly the same distance from a no-slip plane wall, the parallel component of the diffusion tensor governing their interactions decays as $r^{-3}$ rather than $r^{-2}$. This behavior goes undetected in the study of particle pairs by Dufresne et al. ${ }^{30}$ and is observed only in the Stokesian Dynamics of the present work. The leading order contribution to the parallel component of $\mathbf{D}^{\alpha \beta}$ scales as

$$
\left[16 \cos \theta \sin ^{2} \theta \varepsilon_{h}-(3+4 \cos 2 \theta-15 \cos 4 \theta) \varepsilon_{h}^{2}\right] r^{-1},
$$

with an error proportional to $\varepsilon_{h}^{3} r^{-1}$, and in which $\theta$ is the angle made by the line connecting the particles with the normal to the wall and $\varepsilon_{h}=h / r$. When $\theta=\pi / 2$, the particles are the same distance from the wall and the diffusive coupling between the particles scales as $\varepsilon_{h}^{2} r^{-1}$ or equivalently as $r^{-3}$. However, for $\theta=$ $\pi / 2+\varepsilon_{\theta}$ the particles are not at the same height above the wall so that

$$
\left(\mathbf{I}-\mathbf{e}_{\perp} \mathbf{e}_{\perp}\right): \mathbf{D}^{\alpha \beta} \sim\left[-\varepsilon_{\theta} \varepsilon_{h}+\varepsilon_{h}^{2}+O\left(\varepsilon_{\theta}^{3} \varepsilon_{h}, \varepsilon_{\theta}^{2} \varepsilon_{h}^{2}\right)\right] r^{-1} .
$$

In order to detect the $O\left(r^{-3}\right)$ scaling, the conditions: $a \ll h, \varepsilon_{h}$ $\ll 1$ and $\varepsilon_{\theta} \ll \varepsilon_{h}$ must be satisfied. While the quantity $\varepsilon_{h}$ is controlled by the experimental parameters (i.e. the height above the wall and the inter-particle separation are imposed), $\varepsilon_{\theta}$ is the result of experimental error. In fact, $\varepsilon_{\theta}$ is set primarily by fluctuations in the distances of the particles from the wall so that,

$$
\varepsilon_{\theta} a \geq 2 \sqrt{\left\langle\left(h^{\prime}-h\right)^{2}\right\rangle},
$$

where $h^{\prime}-h$ is the deviation of the height of a particle from the imposed height. One contribution to this deviation is the lag time used in tracking the particle trajectories so that $\varepsilon_{h} \geq 2 \sqrt{D_{\perp} \tau} / a$ (this alone is more than $10 \%$ in the present experiments). Other more surreptitious sources of error include the strength and depth of the potential well created by the laser tweezers, the precision and accuracy of the microscopic focal plane normal to the wall and the mean drift of Brownian particles away from the wall. The challenge then is to minimize the fluctuations in the positions of the particles above the wall to such a degree that the $O\left(r^{-3}\right)$ decay of the hydrodynamic interactions is detectable. Studies of suspensions confined to channels only one particle diameter in width have observed this decay rate explicitly. ${ }^{13}$ The effect of such strong confinement is to restrict the particles to residing on the centerline of the channel alone ensuring that $\varepsilon_{h} \approx$ 0 . Controlling the level of unconfined particles is more difficult, though this is a standard by which the accuracy of future experiments similar to those herein may be judged.

\section{Acknowledgements}

We thank A. N. Beris for helpful discussions. We acknowledge the financial support of the NSF (NIRT CBET-0506701).

\section{References}

1 J. F. Brady and G. Bossis, Annu. Rev. Fluid Mech., 1988, 20, 111.

2 G. D. M. MacKay and S. G. Mason, J. Colloid Sci., 1961, 16, 632635.

3 G. E. Charles and S. G. Mason, J. Colloid Sci., 1960, 15, 105-122.

4 G. Barnocky and R. H. Davis, Phys. Fluids, 1988, 31, 1324-1329.

5 C. E. Heath, S. Feng, J. P. Day, A. L. Graham and M. S. Ingber, Phys. Rev. E: Stat., Nonlinear, Soft Matter Phys., 2008, 77, 026307. 
6 G. K. Batchelor, J. Fluid Mech., 1976, 74, 1-29.

7 N. Garnier and N. Ostrowsky, J. Phys. II, 1991, 1(10), 1221-1232.

8 J. Santana-Solano, A. Ram'rez-Saito and J. L. Arauz-Lara, Phys. Rev. Lett., 2005, 95, 198301

9 R. D. Leonardo, S. Keen, F. Ianni, J. Leach, M. J. Padgett and G. Ruocco, Phys. Rev. E: Stat., Nonlinear, Soft Matter Phys., 2008, 78, 031406 .

10 S. M. Anthony, M. Kim and S. Granick, J. Chem. Phys., 2008, 129, 244701.

11 M. Baron, J. Blawzdziewicz and E. Wajnryb, Phys. Rev. Lett., 2008, 100, 174502

12 V. N. Michailidou, G. Petekidis, J. W. Swan and J. F. Brady, Phys. Rev. Lett., 2009, 102, 068302.

13 B. Cui, H. Diamant, B. Lin and S. A. Rice, Phys. Rev. Lett., 2004, 92, 258301

14 A. Pralle, E. L. Florin, E. H. K. Stelzer and J. K. H. Horber, Appl. Phys. A: Mater. Sci. Process., 1998, 66, S71.

15 L. P. Faucheux and A. J. Libchaber, Phys. Rev. E: Stat. Phys., Plasmas, Fluids, Relat. Interdiscip. Top., 1994, 49, 5158.

16 B. Lin, J. Yu and S. A. Rice, Phys. Rev. E: Stat. Phys., Plasmas, Fluids, Relat. Interdiscip. Top., 2000, 62, 3909.

17 S. H. Behrens, J. Plewa and D. G. Grier, Eur. Phys. J. E, 2003, 10, 115-121.

18 J. Leach, H. Mushfique, S. Keen, R. D. Leonardo, G. Ruocco, J. M. Cooper and M. J. Padgett, Phys. Rev. E: Stat., Nonlinear, Soft Matter Phys., 2009, 79, 026301.

19 P. Holmqvist, J. K. G. Dhont and P. R. Lang, J. Chem. Phys., 2007, 126, 044707.
20 H. Faxén, Ark. Mat., Astron. Fys., 1924, 18, 1.

21 C. Pozrikidis, Boundary Integrals and Singularity Methods for Linearized Viscous Flow, Cambridge University Press, New York, 1992.

22 J. Happel and H. Brenner, Low Reynolds Number Hydrodynamics, Kluwer, Dordrecht, 1991.

23 J. W. Merrill, S. K. Sainis, J. Blawzdziewicz and E. R. Dufresne, Soft Matter, 2010, 6, 2187-2192.

24 R. D. Leonardo, S. Keen, J. Leach, C. D. Saunter, G. D. Love, G. Ruocco and M. J. Padgett, Phys. Rev. E: Stat., Nonlinear, Soft Matter Phys., 2007, 76, 061402.

25 J. W. Swan and J. F. Brady, Phys. Fluids, 2007, 19, 113306.

26 S. Kim and S. J. Karilla, Microhydrodynamics: Principles and Selected Applications, Dover, New York, 2005.

27 J. R. Blake, Math. Proc. Cambridge Philos.Soc., 1971, 70, 303310.

28 J. Rotne and S. Prager, J. Chem. Phys., 1969, 50, 4831-4837.

29 R. M. Jendrejack, D. C. Shchwartz, J. J. de Pablo and M. Graham, J. Chem. Phys., 2004, 120, 2513.

30 E. R. Dufresne, T. M. Squires, M. P. Brenner and D. C. Grier, Phys. Rev. Lett., 2000, 85, 3317.

31 J. C. Crocker and D. G. Grier, J. Colloid Interface Sci., 1996, 179, 298-310.

32 L. J. Durlofsky, J. F. Brady and G. Bossis, J. Fluid Mech., 1987, 180, 21-49.

33 P. P. Lele, Directed Self-Assembly in Electrical and Optical Fields, U. Delaware, Ph.D. thesis, 2010. 